\title{
A simple scheme for magnetic balance in four-component relativistic Kohn-Sham calculations of nuclear magnetic resonance shielding constants in a Gaussian basis
}

Cite as: J. Chem. Phys. 136, 014108 (2012); https://doi.org/10.1063/1.3671390

Submitted: 03 November 2011 . Accepted: 01 December 2011 . Published Online: 04 January 2012

Małgorzata Olejniczak, Radovan Bast, Trond Saue, and Magdalena Pecul

\section{ARTICLES YOU MAY BE INTERESTED IN}

A fully relativistic method for calculation of nuclear magnetic shielding tensors with a restricted magnetically balanced basis in the framework of the matrix Dirac-Kohn-Sham equation

The Journal of Chemical Physics 128, 104101 (2008); https://doi.org/10.1063/1.2837472

Gauge origin independent calculations of nuclear magnetic shieldings in relativistic fourcomponent theory

The Journal of Chemical Physics 131, 124119 (2009); https://doi.org/10.1063/1.3240198

Fully relativistic calculations of NMR shielding tensors using restricted magnetically balanced basis and gauge including atomic orbitals

The Journal of Chemical Physics 132, 154101 (2010); https://doi.org/10.1063/1.3359849

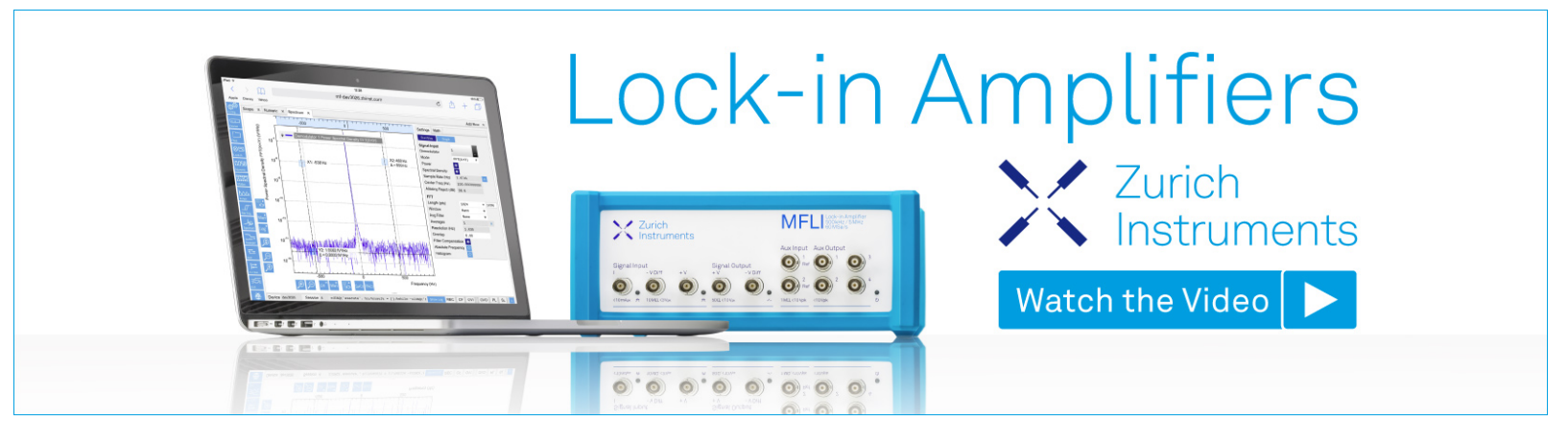




\title{
A simple scheme for magnetic balance in four-component relativistic Kohn-Sham calculations of nuclear magnetic resonance shielding constants in a Gaussian basis
}

\author{
Małgorzata Olejniczak, ${ }^{1}$ Radovan Bast, ${ }^{2,3}$ Trond Saue $^{3}$ and Magdalena Pecull,a) \\ ${ }^{1}$ Department of Chemistry, University of Warsaw, Pasteura 1,02-093 Warsaw, Poland \\ ${ }^{2}$ Centre for Theoretical and Computational Chemistry, Department of Chemistry, University of Troms $\phi$, \\ N-9037 Troms $\phi$, Norway \\ ${ }^{3}$ Laboratoire de Chimie et Physique Quantique (UMR 5626), CNRS/Université de Toulouse 3 (Paul Sabatier), \\ 118 route de Narbonne, 31062 Toulouse, France
}

(Received 3 November 2011; accepted 1 December 2011; published online 4 January 2012)

\begin{abstract}
We report the implementation of nuclear magnetic resonance (NMR) shielding tensors within the four-component relativistic Kohn-Sham density functional theory including non-collinear spin magnetization and employing London atomic orbitals to ensure gauge origin independent results, together with a new and efficient scheme for assuring correct balance between the large and small components of a molecular four-component spinor in the presence of an external magnetic field (simple magnetic balance). To test our formalism we have carried out calculations of NMR shielding tensors for the $\mathrm{HX}$ series $(\mathrm{X}=\mathrm{F}, \mathrm{Cl}, \mathrm{Br}, \mathrm{I}, \mathrm{At})$, the $\mathrm{Xe}$ atom, and the Xe dimer. The advantage of simple magnetic balance scheme combined with the use of London atomic orbitals is the fast convergence of results (when compared with restricted kinetic balance) and elimination of linear dependencies in the basis set (when compared to unrestricted kinetic balance). The effect of including spin magnetization in the description of NMR shielding tensor has been found important for hydrogen atoms in heavy HX molecules, causing an increase of isotropic values of $10 \%$, but negligible for heavy atoms. (C) 2012 American Institute of Physics. [doi:10.1063/1.3671390]
\end{abstract}

\section{INTRODUCTION}

Nuclear magnetic resonance (NMR) shielding constants and spin-spin coupling constants are among the most important spectroscopic parameters used in chemistry and biology, as they provide valuable insight into the electronic structure of systems under study. ${ }^{1}$ The basic (non-relativistic) theory for these parameters was formulated in a series of landmark papers in the 1950s by Ramsey and co-workers ${ }^{2-6}$ and since then non-relativistic $a b$ initio calculations of these parameters have become a routine task. However, these parameters can exhibit strong relativistic effects: first, they are magnetic properties and therefore inherently relativistic phenomena, ${ }^{7}$ and second, the associated property operators probe the electron density in the core region, where relativistic effects are the most pronounced. These effects are non-negligible even in the case of relatively light nuclei like ${ }^{31} \mathrm{P}^{8}$ For the ${ }^{129} \mathrm{Xe}$ nucleus relativistic effects are significant and relativity contributes approximately $20 \%$ to the shielding constant. ${ }^{9}$ For heavy nuclei such as ${ }^{199} \mathrm{Hg}$, relativistic effects are critically important. ${ }^{10}$ Since NMR of heavy spin-1/2 nuclei such as ${ }^{129} \mathrm{Xe},{ }^{183} \mathrm{~W}$, ${ }^{195} \mathrm{Pt}$, and ${ }^{199} \mathrm{Hg}$, is a spectroscopic method of considerable importance, and since the presence of heavy nuclei strongly affects NMR parameters also of neighboring light nuclei, ${ }^{11,12}$ theoretical methods for the modeling of NMR parameters including relativistic effects are in demand. Much effort has

\footnotetext{
a) Author to whom correspondence should be addressed. Electronic mail: mpecul@chem.uw.edu.pl.
}

therefore been put into the development of relativistic methods for modeling the NMR shielding tensor resulting in several implementations, which are reviewed in Refs. 1,13-16.

There are multiple difficulties in the formulation of a correct formalism for calculating shielding tensors. An important challenge stems from the gauge-dependence of results when finite basis sets are employed. ${ }^{17-20}$ One solution to this problem is to employ perturbation-dependent basis functions that shift the gauge origin to their respective center, thereby ensuring that the calculated magnetic properties are independent of the position of the gauge origin. These basis functions are called London atomic orbitals (LAOs) (Refs. 21 and 22) also known in literature as gauge-including atomic orbitals (GIAOs). ${ }^{23}$ Other solutions to the problem of gaugedependence are well established in non-relativistic calculations and have been reviewed for instance in Ref. 24.

The LAO formalism is at least conceptually easily extensible to any combination of wave function ansatz and Hamiltonian, including relativistic Hamiltonians. ${ }^{25-30}$ A difficulty particular to relativistic calculations of magnetic properties originates from the fact that the inclusion of a vector potential affects the balance between the large and the small components of the four-component spinors. This magnetic balance ${ }^{31-33}$ must be taken into account, explicitly or implicitly, in order to obtain correct results for magnetic properties in the relativistic domain.

An important difference between relativistic and nonrelativistic theory is the lack of an explicit diamagnetic term in the Hamiltonian. The non-relativistic Hamiltonian has 
a paramagnetic term (linear in the vector potential) and a diamagnetic term (quadratic in the vector potential). The paramagnetic term contributes to second-order magnetic properties through a linear response function, whereas the non-relativistic diamagnetic term can be evaluated through an expectation value. In contrast, the relativistic Hamiltonian is strictly linear in both electric and magnetic fields. However, it can be shown that the diamagnetic term enters through the contribution of the negative-energy orbitals. ${ }^{31,34,35}$ It should be noted that this is only true in the framework of perturbation theory where the orthogonal complement to the space of occupied positive-energy orbitals serves as a basis for the construction of first- and higher order corrections to the unperturbed orbitals, and is thus not a feature of variational calculations. It should also be noted that the negative-energy orbitals do not disappear in the non-relativistic limit. Instead, the positive and negative solutions become completely decoupled by an infinite energy gap and can be separately expanded in 2-spinor bases. The relativistic expression for the NMR shielding constant thus goes smoothly into its non-relativistic counterpart provided that the speed of light appearing in the vector potential associated with nuclear spin is kept fixed at its relativistic value. ${ }^{7}$

The reason why the contribution from the negativeenergy orbitals is important for magnetic properties and not for electric properties is that magnetic fields are introduced through operators which are odd in the sense that they couple large and small components, whereas electric fields are represented by operators that are even in this respect. Kutzelnigg proposed a field-dependent unitary transformation of the four-component relativistic Hamiltonian in order to introduce an explicit diamagnetic term. ${ }^{33}$ If the vector potentials associated with both external magnetic fields and nuclear spins are included, leading to the so-called full field-dependent unitary transformation, ${ }^{36}$ the response contribution from the negative-energy orbitals is reduced from $\mathcal{O}\left(c^{0}\right)$ to $\mathcal{O}\left(c^{-4}\right)$, and thus disappear in the non-relativistic limit, but numerical instabilities appear. ${ }^{37}$ It is therefore recommended to include only the external field, leading to the so-called external field-dependent unitary transformation ${ }^{36}$ with contributions of $\mathcal{O}\left(c^{-2}\right)$ from negative-energy orbitals. However, in both cases the negative-energy orbitals cannot be neglected in relativistic calculations of magnetic properties. ${ }^{38}$

There have been several earlier attempts to calculate NMR shielding tensor in a four-component formalism by the Nakatsuji group ${ }^{39-42}$ and by Visscher et al. ${ }^{43}$ However, these implementations have employed a common gauge origin (CGO) approach. A first implementation of London orbitals, albeit incomplete, was reported by Quiney and co-workers. ${ }^{25,44}$ The first consistent implementation of the shielding tensor with LAOs at the four-component relativistic Hartree-Fock (HF) level has been carried out within the DIRAC program ${ }^{45}$ by one of the authors (T.S.) (Ref. 27) and applied to several systems. ${ }^{46-48}$ The use of LAOs at the fourcomponent HF level has also been reported by Hamaya and Fukui. ${ }^{28}$ The first four-component formalism for NMR properties using density functional theory (DFT) methods was presented by Komorovský et al. ${ }^{30}$ within the ReSpect code, ${ }^{49}$ where a matrix formulation of four-component relativistic
Kohn-Sham (KS) equations with restricted magnetic balance (mDKS-RMB) and with GIAOs was proposed. A GIAO implementation within the BDF package was recently reported by Cheng et $_{\text {al. }}{ }^{29}$

In the present work, we report the implementation of LAOs in four-component relativistic DFT including noncollinear spin magnetization (known as spin-density functional theory, SDFT) for the purpose of calculating NMR shielding tensors. A new efficient scheme, which we call simple magnetic balance (sMB), for handling the balance between large and small components of the molecular spinor is also presented. Our formulation is in many aspects similar to the one proposed by Komorovský et al. ${ }^{38}$ except that our approach is fully analytical and more flexible in the choice of exchange-correlation (XC) functionals.

The implementation has been tested by carrying out calculations of NMR shielding tensors for the HX series $(\mathrm{X}=\mathrm{F}$, $\mathrm{Cl}, \mathrm{Br}, \mathrm{I}, \mathrm{At})$. This is a well established test set for relativistic calculations of NMR properties ${ }^{9,50}$ and allows for a comparison with the values obtained by means of the mDKS-RMBGIAO scheme of Komorovský et al. ${ }^{30}$ We have also compared our results with an earlier work by the same group using CGO (Ref. 38) since the authors discuss differences between "coupled" and "uncoupled" approximations, which for non-hybrid functionals corresponds to the DFT and SDFT approaches reported here.

Our other test systems are the Xe atom and Xe dimer. This choice has been motivated by the availability of results from the Vaara group, $,, 46,50$ where relativity is included by perturbational corrections, as well as non-relativistic results by Jameson et $_{\text {al. }}{ }^{51}$

Our article is organized as follows: in Sec. II we present the theoretical formulation of the implementation, focusing on the simple scheme to obtain the magnetic balance and on the XC contributions to the linear response equations in perturbation-dependent basis sets. The computational details are described in Sec. III, while the results of test calculations are presented in Sec. IV. Conclusions are given in Sec. V.

\section{THEORY}

The central quantity in this work is the electronic contribution to the NMR shielding tensor, $\sigma_{\alpha \beta}^{A}$, which in the BornOppenheimer approximation can be calculated as the secondorder derivative of the electronic energy with respect to the external magnetic field $\vec{B}$ and the nuclear magnetic moment $\vec{M}^{A}$ at nucleus $A$ at zero field strength for the magnetic field and all nuclear magnetic moments $\vec{M}^{K}$ (indicated by subscript "0"):

$$
\sigma_{\alpha \beta}^{A}=\left.\frac{\mathrm{d}^{2} E}{\mathrm{~d} B_{\alpha} \mathrm{d} M_{\beta}^{A}}\right|_{0} .
$$

In this section we will first point out that the introduction of LAOs makes magnetic balance atomic, such that it is possible to obtain (unrestricted) magnetic balance by extending the orbitals obtained in a self-consistent field (SCF) calculation using restricted kinetic balance (RKB) by their unrestricted kinetic balance (UKB) complement. We next detail 
how the previously reported four-component relativistic LAO implementation ${ }^{27}$ is extended from HF calculations to KS SDFT calculations. We will therefore focus on the new contributions to the calculated property due to the XC terms. For a more detailed discussion of response theory in perturbationdependent basis we refer the reader to Sec. II of Ref. 27.

In the following, the Einstein convention of summation over repeated indices is adopted. Indices $i, j, \ldots$ are used for occupied molecular orbitals, indices $a, b, \ldots$ for virtual orbitals and indices $p, q, \ldots$ for orbitals in general. Greek indices are used for the three Cartesian components and Latin indices are used for the components of four-component vector. We employ SI-based atomic units.

\section{A. Magnetic balance and LAOs}

Our starting point is the four-component relativistic KS equation

$$
\left[\begin{array}{cc}
V \mathbf{I}_{2 \times 2} & c(\overrightarrow{\boldsymbol{\sigma}} \cdot \vec{p}) \\
c(\overrightarrow{\boldsymbol{\sigma}} \cdot \vec{p}) & \left(V-2 m c^{2}\right) \mathbf{I}_{2 \times 2}
\end{array}\right]\left[\begin{array}{l}
\psi^{\mathrm{L}} \\
\psi^{\mathrm{S}}
\end{array}\right]=\left[\begin{array}{l}
\psi^{\mathrm{L}} \\
\psi^{\mathrm{S}}
\end{array}\right] E,
$$

where appears the speed of light $c$, the electron mass $m$, the vector of $2 \times 2$ Pauli spin matrices $\vec{\sigma}$, and the $2 \times 2$ identity matrix $\mathbf{I}_{2 \times 2}$. The operator $V=-e \phi$ describes the interaction with the KS scalar potential $\phi$ and can be decomposed into contributions from the classical Coulomb potential of nuclei $V_{\mathrm{N}}$ and electrons $V_{\mathrm{H}}$ as well as the (approximate) $\mathrm{XC}$ potential $V_{\mathrm{xc}}$ (the extension to hybrid functionals is straightforward). Most finite basis approximations to this equation employ RKB between individual large (L) and small (S) basis functions based on the coupling between the large and small components in the non-relativistic limit (NRL)

$$
\lim _{c \rightarrow \infty} c \psi^{\mathrm{S}}=\frac{1}{2 m}(\overrightarrow{\boldsymbol{\sigma}} \cdot \vec{p}) \psi^{\mathrm{L}} .
$$

Kutzelnigg has demonstrated that for relativistic hydrogenlike atoms the $1 s$-orbital can be expanded to completeness in an even-tempered kinetically balanced Gaussian basis with convergence characteristics similar to that of the nonrelativistic case. ${ }^{52}$ In practice, however, a finite-size basis set must have sufficient flexibility such that the exact relativistic coupling of the large and small components can be obtained and/or atomic balance should be employed. ${ }^{53}$

One way to implement RKB is to start from twocomponent large component basis functions of Gaussian type

$$
{ }^{2 c} G_{n, \kappa, m}\left(\vec{r}_{A}\right)=N_{n-1} r_{A}^{n-1} \exp \left[-\alpha r_{A}^{2}\right] \zeta_{\kappa, m}\left(\theta_{A}, \phi_{A}\right),
$$

where $\vec{r}_{A}=\vec{r}-\vec{R}_{A}$, the index $A$ referring to the center of expansion, and $\zeta_{\kappa, m}$ are the two-component atomic angular functions. The principal quantum number $n$ is by convention set to $n=l+1$ such that the RKB prescription gives

$$
\begin{aligned}
(\overrightarrow{\boldsymbol{\sigma}} \cdot \vec{p}){ }^{2 c} G_{l+1, \kappa, m}\left(\vec{r}_{A}\right)= & N_{l}\left[(\kappa+l+1) r_{A}^{l-1}-2 \alpha r_{A}^{l+1}\right] \\
& \times \exp \left[-\alpha r_{A}^{2}\right] \zeta_{-\kappa, m}\left(\theta_{A}, \phi_{A}\right) .
\end{aligned}
$$

Starting from a large component basis function ${ }^{2 c} G_{l+1, \kappa, m}$, a single Gaussian basis function ${ }^{2 c} G_{l+2,-\kappa, m}$ is obtained for negative $\kappa=-(l+1)$, whereas positive $\kappa=l$ leads to a non-standard function $\left[(2 l+1)-2 \alpha r^{2}\right]^{2 c} G_{l,-\kappa, m}$. RKB can equivalently be obtained by transformation to the modified Dirac equation at the operator level: ${ }^{38,54-56}$

$$
\left[\begin{array}{c}
\psi^{\mathrm{L}} \\
\psi^{\mathrm{S}}
\end{array}\right]=\left[\begin{array}{cc}
\mathbf{I}_{2 \times 2} & \mathbf{0}_{2 \times 2} \\
\mathbf{0}_{2 \times 2} & \frac{1}{2 m c}(\overrightarrow{\boldsymbol{\sigma}} \cdot \vec{p})
\end{array}\right]\left[\begin{array}{c}
\psi^{\mathrm{L}} \\
\phi^{\mathrm{L}}
\end{array}\right]=E \tilde{\psi}
$$

This approach has the advantage that the large $\psi^{\mathrm{L}}$ and pseudo-large $\phi^{\mathrm{L}}$ components can be expanded in the same basis, but on the other hand this approach requires the programming of new integrals. A third approach to RKB is to carry out the transformation to the modified Dirac equation at the matrix level. ${ }^{57}$ One may then start from an expansion of the individual components of the Dirac 4-spinor in scalar basis functions known from the non-relativistic domain, that is, Cartesian Gaussian-type basis functions

$$
G_{i j k}^{A}=N_{l} x_{A}^{i} y_{A}^{j} z_{A}^{k} \exp \left[-\alpha r_{A}^{2}\right] .
$$

Starting from the large component set

$$
G_{l}^{A}=\left\{G_{i j k}^{A}\right\} ; \quad \forall i+j+k=l,
$$

the small component set $\nabla G_{l}^{A}=\left\{G_{l-1}^{A}, G_{l+1}^{A}\right\}$ is generated by UKB. The subsequent procedure can be represented schematically as

$$
\begin{aligned}
& \mathbf{F}_{0} \mathbf{c}_{0}=\mathbf{S}_{0} \mathbf{c}_{0} \varepsilon \\
& \text { (i) } \\
& \mathbf{F}_{1} \mathbf{c}_{1}=\mathbf{c}_{1} \varepsilon \\
& \text { (ii) } \quad \downarrow \mathbf{S}_{2}=\mathbf{W}^{\dagger} \mathbf{W} \\
& \mathbf{F}_{2} \mathbf{c}_{2}=\mathbf{S}_{2} \mathbf{c}_{2} \varepsilon \\
& \text { (iii) } \quad \downarrow \mathbf{V}_{2}^{\dagger} \mathbf{S}_{2} \mathbf{V}_{2}=\mathbf{I} \\
& \mathbf{F}_{3} \mathbf{c}_{3}=\mathbf{c}_{3} \varepsilon
\end{aligned}
$$

In this procedure the KS matrix is first set up in the nonorthogonal UKB basis and transformed to orthonormal basis as $\mathbf{F}_{1}=\mathbf{V}_{1}^{\dagger} \mathbf{F}_{0} \mathbf{V}_{1}$. The transformation to the modified Dirac equation is then carried out using a matrix representation of the operator $\hat{W}$ of Eq. (6). Finally, orthonormality is restored by the transformation $\mathbf{V}_{2}$. In practice, the three transformations are combined as $\mathbf{V}=\mathbf{V}_{1} \mathbf{W} \mathbf{V}_{2}$.

We will now turn to a discussion of the kinetic balance in the presence of magnetic perturbations. Magnetic fields are introduced according to the principle of minimal electromagnetic substitution ${ }^{58,59}$

$$
\vec{p} \rightarrow \vec{\pi}=\vec{p}+e \vec{A},
$$

where $\vec{\pi}$ is the mechanical momentum and $\vec{A}$ the vector potential. In the presence of a vector potential the coupling between the large and the small components is modified, giving RMB (Refs. 31-33) in the NRL

$$
\lim _{c \rightarrow \infty} c \psi^{\mathrm{S}}=\frac{1}{2 m}(\overrightarrow{\boldsymbol{\sigma}} \cdot \vec{\pi}) \psi^{\mathrm{L}} .
$$

In the following, we consider the vector potential associated with an external homogeneous magnetic field

$$
\vec{A}_{G}(\vec{r})=\frac{1}{2} \vec{B} \times \vec{r}_{G} ; \quad \vec{r}_{G}=\vec{r}-\vec{R}_{G},
$$

where $\vec{R}_{G}$ is the chosen gauge origin. It is easily shown that RKB does not guarantee RMB. A simple example is provided by the helium atom: ${ }^{47} \mathrm{RKB}$ generates a $p_{1 / 2}$-type small 
component function from the large $s_{1 / 2}$ component, whereas RMB requires in addition a $p_{3 / 2}$ function which in a RKB framework is only obtained by adding a $d_{3 / 2}$ large component function to the basis, thus going beyond basis sets typically employed for calculating the energy and even shieldings. RMB can be implemented explicitly at the operator level, as reported by Komorovský et al. ${ }^{30}$ More generally, as emphasized by Liu and co-workers ${ }^{36}$ various schemes can be devised for the construction of the complement to RKB needed for the construction of the first-order correction to the reference wave functions. All such schemes require the programming of new integrals, but, once implemented, lead to efficient calculation of NMR shieldings in a relativistic framework. Here, we consider a much simpler approach, which requires no new integrals.

Starting from the large component set, Eq. (8), the small component set generated by unrestricted magnetic balance (UMB) is

$$
\left(\vec{p}+e \vec{A}_{G}\right) G_{l}^{A}=\left\{G_{l-1}^{A}, G_{l+1}^{A}\right\} \cup\left\{\vec{r}_{G} G_{l}^{A}\right\} .
$$

The UMB complement $\left\{\vec{r}_{G} G_{l}^{A}\right\}$ to the UKB set $\left\{G_{l-1}^{A}, G_{l+1}^{A}\right\}$ is spanned by the latter only in the atomic case for which it is natural to set $G=A$. In the general molecular case there is, however, no obvious choice of the CGO. The situation changes with the introduction of LAOs

$$
\omega^{A}=\exp \left[-\mathrm{i} \vec{A}_{G}\left(\vec{R}_{A}\right) \cdot \vec{r}\right] \chi^{A} .
$$

We now have

$$
\left(\vec{p}+e \vec{A}_{G}\right) \omega^{A}=\exp \left[-\mathrm{i} \vec{A}_{G}\left(\vec{R}_{A}\right) \cdot \vec{r}\right]\left(\vec{p}+e \vec{A}_{A}\right) \chi^{A}
$$

such that the gauge origin is shifted from arbitrary $\left(\vec{R}_{G}\right)$ to the atomic origin $\left(\vec{R}_{A}\right)$ of the basis function. Of great importance in a relativistic context is that the magnetic balance, Eq. (11), now becomes atomic ${ }^{27}$ such that UMB is fully spanned by the UKB set, Eq. (13). On the other hand UKB generally leads to a small component basis larger than the large component basis set from which it was generated and may increase computational cost as well as introduce linear dependencies in the basis set as compared to RKB. However, such disadvantages are circumvented by a simple scheme: we propose to optimize the zeroth-order orbitals using RKB according to the scheme outlined by Eq. (9) and then extend the RKB coefficients by the UKB complement for use in subsequent calculations of NMR shieldings based on UKB in combination with LAOs. The UKB calculations employ the orthonormal basis defined by transformation ( $i$ ) of Eq. (9). The RKB coefficients are read in and transformed to orthonormal basis using $\mathbf{V}_{1}^{-1}=\mathbf{V}_{1}^{\dagger} \mathbf{S}_{0}$. They are then projected out of the transformation matrix $\mathbf{V}_{1}$ in orthonormal basis; the latter is simply the identity matrix. The remainder constitutes the UKB complement and is transformed back to the AO basis using $\mathbf{V}_{1}$ and added to the RKB coefficients. We emphasize that working in orthonormal basis also has the advantage that linear dependencies are eliminated by the use of a threshold on eigenvalues of the overlap matrix $\mathbf{S}_{0}$. We associate orbital energies $-2 m c^{2}$ with these additional coefficients, which is a good approximation since their large components are strictly zero. The extension of RKB coefficients by the UKB complement has negligible computational cost and, as we will see in Sec. IV, reproduces very well results of more elaborate schemes for the incorporation of magnetic balance.

\section{B. Density and spin density derivatives in the LAO basis}

We now turn to the theory for the calculation of the NMR shielding tensor at the four-component relativistic DFT level using LAOs. The present formulation builds on previous implementations of four-component relativistic DFT, ${ }^{60}$ linear response at the HF (Ref. 61) and DFT (Ref. 62) levels as well as time-dependent DFT (TD-DFT) with non-collinear spin magnetization. ${ }^{63}$ The fundamental variables in this approach are the (number) density $\rho_{0}$, and the spin magnetization vector with the components $\rho_{\mu}(\mu=x, y, z)$. The general density component will be denoted as $\rho_{k}$, where $k=0, x, y, z .^{38,63}$

We employ an exponential parametrization of the KS determinant ${ }^{60,64}$

$$
|\tilde{0}\rangle=\exp (-\hat{\kappa})|0\rangle
$$

which allows for a straightforward identification of redundant variational parameters and the use of unconstrained optimization techniques. In the following, we will limit our attention to a closed-shell reference. In the absence of LAOs the full response of the system is carried by the elements of the anti-Hermitian orbital rotation matrix $\boldsymbol{\kappa}$ where the redundant occupied-occupied and virtual-virtual blocks are set to zero. We accordingly write the orbital rotation operator $\hat{\kappa}$ as

$$
\hat{\kappa}=\kappa_{a i} \hat{a}^{\dagger} \hat{i}-\kappa_{a i}^{*} \hat{i}^{\dagger} \hat{a} .
$$

The general density component is given by

$$
\rho_{k}(\vec{r}, \vec{B})=\breve{\Omega}_{p q ; k}(\vec{r}, \vec{B}) \tilde{D}_{p q}(\kappa) ; \quad k \in\{0, x, y, z\},
$$

where appears the generalized overlap distributions in LAO basis:

$\breve{\Omega}_{p q ; k}=\breve{\psi}_{p}^{\dagger} \boldsymbol{\Sigma}_{k} \breve{\psi}_{q} ; \quad \boldsymbol{\Sigma}_{0}=\mathbf{I}_{4 \times 4}, \quad \boldsymbol{\Sigma}_{\mu}=\left[\begin{array}{cc}\boldsymbol{\sigma}_{\mu} & \mathbf{0}_{2 \times 2} \\ \mathbf{0}_{2 \times 2} & \boldsymbol{\sigma}_{\mu}\end{array}\right]$.

The density component $\rho_{k}$ is therefore both explicitly $(\vec{B})$ and implicitly $(\boldsymbol{\kappa})$ field-dependent. The notation $\breve{A}$ is used for quantities expanded in orthonormal LAO basis. The perturbation-dependent molecular orbitals are expressed as

$$
\breve{\psi}_{p}=\psi_{q}^{\mathrm{UMO}} T_{q p} ; \quad \psi_{q}^{\mathrm{UMO}}=\omega_{\mu} c_{\mu q},
$$

where $\psi_{q}^{\mathrm{UMO}}$ is the unmodified molecular orbital expanded in the set $\left\{\omega_{\mu}\right\}$ of LAOs. The expansion coefficients $\left\{c_{\mu q}\right\}$ are those of the unperturbed orbitals, which leads to the introduction of a field-dependent connection matrix $\mathbf{T}$ to guarantee orthonormality of orbitals at all field strengths. There is a freedom of choice for the connection, but we have employed the recommended natural connection ${ }^{65}$ in the present work. It is easily shown that all connections satisfy

$$
\mathbf{T}^{\dagger B_{\alpha}}+\mathbf{T}^{B_{\alpha}}=-\mathbf{S}^{B_{\alpha}},
$$

where $\mathbf{S}$ is the field-dependent overlap matrix expressed in terms of the unmodified molecular orbitals $\psi_{p}^{\mathrm{UMO}}$ and the 
superscript " $B_{\alpha}$ " denotes differentiation with respect to the magnetic field component $B_{\alpha}$ at zero field:

$$
\mathbf{T}^{B_{\alpha}}=\left.\frac{\mathrm{d}}{\mathrm{d} B_{\alpha}} \mathbf{T}\right|_{0} ; \quad \mathbf{S}^{B_{\alpha}}=\left.\frac{\mathrm{d}}{\mathrm{d} B_{\alpha}} \mathbf{S}\right|_{0} .
$$

The computationally useful formula for $\rho_{k}$ is obtained by applying the Baker-Campbell-Hausdorff expansion of the density matrix:

$$
\tilde{D}_{p q}(\kappa)=\left\langle\tilde{0}\left|\hat{p}^{\dagger} \hat{q}\right| \tilde{0}\right\rangle=\left\langle 0\left|\hat{p}^{\dagger} \hat{q}\right| 0\right\rangle+\left\langle 0\left|\left[\hat{\kappa}, \hat{p}^{\dagger} \hat{q}\right]\right| 0\right\rangle+\mathcal{O}\left(\kappa^{2}\right),
$$

which allows us to rewrite $\rho_{k}$ as a series expansion in terms of the orbital rotation matrix $\boldsymbol{\kappa}$

$$
\rho_{k}=\breve{\Omega}_{i i ; k}^{(0)}+\breve{\Omega}_{i i ; k}^{(1)}+\frac{1}{2 !} \breve{\Omega}_{i i ; k}^{(2)}+\cdots=\sum_{n=0}^{\infty} \frac{1}{n !} \breve{\Omega}_{i i ; k}^{(n)},
$$

where, following Sałek et al. ${ }^{62}$ we have introduced the recurrence formula for the generalized $n$-fold one-index transformed overlap distribution: ${ }^{62}$

$$
\breve{\Omega}_{p q ; k}^{(n)}=\kappa_{p t} \breve{\Omega}_{t q ; k}^{(n-1)}-\breve{\Omega}_{p t ; k}^{(n-1)} \kappa_{t q} ; \quad \breve{\Omega}_{p q ; k}^{(0)}=\breve{\psi}_{p}^{\dagger} \Sigma_{k} \breve{\psi}_{q} .
$$

In Sec. II C we will require the following derivatives of $\rho_{k}$ with respect to orbital rotation parameters and with respect to components of the external magnetic field to construct the $\mathrm{XC}$ contributions to the linear response equations according to the chain differentiation rule:

1. The derivative of $\rho_{k}$ with respect to the $\alpha$-component of the external magnetic field:

$$
\left.\frac{\partial \rho_{k}}{\partial B_{\alpha}}\right|_{0}=\Omega_{i i ; k}^{B_{\alpha}}
$$

2. The derivative of the density with respect to the orbital rotation amplitudes:

$$
\left.\frac{\partial \rho_{k}}{\partial \kappa_{a i}}\right|_{0}=-\Omega_{i a ; k}
$$

3. The mixed derivatives:

$$
\left.\frac{\partial^{2} \rho_{k}}{\partial B_{\alpha} \partial \kappa_{a i}}\right|_{0}=-\Omega_{i a ; k}^{B_{\alpha}}
$$

The above expressions contain explicit derivatives of the overlap distributions with respect to the magnetic field component $B_{\alpha}$

$\Omega_{p q ; k}^{B_{\alpha}}=\frac{\mathrm{i}}{2}\left(\vec{R}_{M N} \times \vec{r}\right)_{\alpha} c_{\mu p}^{*} c_{\nu q} \Omega_{\mu v ; k}+T_{t p}^{* B_{\alpha}} \Omega_{t q ; k}+\Omega_{p t ; k} T_{t q}^{B_{\alpha}}$,

where $\vec{R}_{M N}=\vec{R}_{M}-\vec{R}_{N}$ is the distance vector between the centers of atomic orbitals $\chi_{\mu}$ and $\chi_{\nu}$, for which the generalized overlap is given by $\Omega_{\mu v ; k}=\chi_{\mu}^{\dagger} \Sigma_{k} \chi_{\nu}$.

The density derivatives presented above have no explicit reference to the gauge origin which is the desired feature of a gauge-origin independent implementation. In particular, the direct contribution originating from the differentiation of the London phase factor with respect to magnetic field (which is the first term of Eq. (29)) depends only on the distance between two centers of atomic orbitals. In the non-relativistic framework $\rho_{0}$ does not contribute to the direct term since $\vec{R}_{M N} \times \vec{r}$ is antisymmetric with respect to the permutation of indices $\mu$ and $\nu$ (positions of centers $M$ and $N$ ), while $\Omega_{\mu \nu ; 0}$ is symmetric. In the relativistic case this derivative term is zero due to time-reversal symmetry.

\section{Four-component relativistic DFT linear response with LAOs}

At the SCF level the elements of the NMR shielding tensor can be expressed as the first-order correction to the expectation value of the hyperfine Hamiltonian

$$
\hat{h}_{M^{A}}=\frac{1}{c^{2} r_{A}^{3}}\left(\vec{r}_{A} \times c \overrightarrow{\boldsymbol{\alpha}}\right)
$$

due to an external magnetic field, that is,

$$
\sigma_{\alpha \beta}^{A}=\left\{\left\langle\tilde{\psi}_{i}^{B_{\alpha}}\left|\hat{h}_{M_{\beta}^{A}}\right| \psi_{i}\right\rangle+\left\langle\psi_{i}\left|\hat{h}_{M_{\beta}^{A}}\right| \tilde{\psi}_{i}^{B_{\alpha}}\right\rangle\right\} .
$$

With the parametrization introduced in Sec. II B the first-order occupied KS orbitals are given as

$$
\tilde{\psi}_{i}^{B_{\alpha}}=\left.\frac{\mathrm{d} \tilde{\psi}_{i}}{\mathrm{~d} B_{\alpha}}\right|_{0}=\omega_{\mu}^{B_{\alpha}} c_{\mu i}+\psi_{q} T_{q i}^{B_{\alpha}}-\psi_{a} \kappa_{a i}^{B_{\alpha}} .
$$

The first-order orbital rotation amplitudes are collected in the solution vector $\mathbf{X}_{B_{\alpha}}$ and obtained by solving the linear response equation

$$
\mathbf{E}_{0}^{[2]} \mathbf{X}_{B_{\alpha}}=-\mathbf{E}_{B_{\alpha}}^{[1]}
$$

where $\mathbf{E}_{0}^{[2]}$ is the electronic Hessian and $\mathbf{E}_{B_{\alpha}}^{[1]}$ is the (generalized) property gradient. In Ref. 27 the authors demonstrated that the only quantity that needs to be modified due to the introduction of perturbation-dependent orbitals at the HF level is the property gradient:

$$
\mathbf{E}_{B_{\alpha}}^{[1]}=\left[\begin{array}{c}
\mathbf{g}^{B_{\alpha}} \\
\mathbf{g}^{* B_{\alpha}}
\end{array}\right] ; \quad g_{a i}^{B_{\alpha}}=-\tilde{F}_{a i}^{B_{\alpha}},
$$

where $\tilde{F}_{a i}^{B_{\alpha}}$ is the derivative of the Fock matrix in orthogonalized MO basis with respect to the external magnetic field component $B_{\alpha}$, here written in a slightly modified form with respect to Ref. 27,

$$
\begin{aligned}
\tilde{F}_{p q}^{B_{\alpha}}= & h_{p q ; 0}^{B_{\alpha}}+\left[h_{p q}^{B_{\alpha}}+\mathcal{L}_{p q j j}^{\gamma B_{\alpha}}\right]+\left[T_{t j}^{* B_{\alpha}} \mathcal{L}_{p q t j}^{\gamma}+\mathcal{L}_{p q j t}^{\gamma} T_{t j}^{B_{\alpha}}\right] \\
& +\left[T_{t p}^{* B_{\alpha}} F_{t q ; k}+F_{p t ; k} T_{t q}^{B_{\alpha}}\right] .
\end{aligned}
$$

Two-electron integrals enter through $\mathcal{L}_{p q r s}^{\gamma}=(p q \mid r s)$ $-\gamma(p s \mid r q)$, where $\gamma$ represents the amount of orbital exchange. For HF $\gamma=1$, for non-hybrid XC functionals $\gamma=0$ and for hybrid XC functionals $0<\gamma<1$. After this rearrangement, we can easily distinguish four different contributions to the property gradient: the first term, $h_{p q ; 0}^{B_{\alpha}}$, is the fieldindependent property gradient, the second, $\left[h_{p q}^{B_{\alpha}}+\mathcal{L}_{p q j j}^{\gamma B_{\alpha}}\right]$, is a Fock matrix constructed from one- and two-electron integrals modified by the presence of LAOs, ${ }^{27}$ the third, $\left[T_{j t}^{* B_{\alpha}} \mathcal{L}_{p q t j}^{\gamma}\right.$ $\left.+\mathcal{L}_{p q j t}^{\gamma} T_{t j}^{B_{\alpha}}\right]$, is a two-electron Fock matrix constructed from a density matrix modified by reorthonormalization, and the last term, $\left[T_{t p}^{* B_{\alpha}} F_{t q ; k}+F_{p t ; k} T_{t q}^{B_{\alpha}}\right]$, is the one-index 
transformed Fock matrix with respect to the first-order connection matrix derivative $\mathbf{T}^{\mathbf{B}_{\alpha}}$.

Introducing the $\mathrm{XC}$ contribution to the property gradient requires the explicit implementation of the second and third terms in Eq. (35), while the last term in Eq. (35) is straightforwardly obtained by replacing the Fock matrix by the KS matrix. Generally, the XC contribution to the property gradient can be written as:

$$
\begin{aligned}
\left.\frac{\partial}{\partial B_{\alpha}} \frac{\partial E_{\mathrm{xc}}}{\partial \kappa_{p q}}\right|_{0}= & \left.\left.\iint \frac{\delta^{2} E_{\mathrm{xc}}}{\delta \rho_{k}\left(\vec{r}_{1}\right) \delta \rho_{k^{\prime}}\left(\vec{r}_{2}\right)} \frac{\partial \rho_{k}\left(\vec{r}_{1}\right)}{\partial \kappa_{p q}}\right|_{0} \frac{\partial \rho_{k^{\prime}}\left(\vec{r}_{2}\right)}{\partial B_{\alpha}}\right|_{0} \mathrm{~d} \vec{r}_{1} \mathrm{~d} \vec{r}_{2} \\
& +\left.\int \frac{\delta E_{\mathrm{xc}}}{\delta \rho_{k}\left(\vec{r}_{1}\right)} \frac{\partial^{2} \rho_{k}\left(\vec{r}_{1}\right)}{\partial \kappa_{p q} \partial B_{\alpha}}\right|_{0} \mathrm{~d} \vec{r}_{1} .
\end{aligned}
$$

Here we use functional derivatives of the XC energy, but since approximate functionals typically employ a local ansatz, in which the energy density is a function of local density variables, the computationally useful expressions are obtained by replacing functional derivatives by function derivatives: for local density approximation (LDA) functionals it is an easy replacement, whereas the GGA working equations can be obtained either using integration by parts or by calculating the above derivatives from an explicit expression of the integrand of $E_{\mathrm{xc}}$. An immediate consequence of such a local ansatz is that the double integral in Eq. (36) can be reduced to onedimensional integral, hence

$$
\begin{aligned}
\left.\frac{\partial}{\partial B_{\alpha}} \frac{\partial E_{\mathrm{xc}}}{\partial \kappa_{p q}}\right|_{0}= & \left.\left.\int \frac{\delta^{2} E_{\mathrm{xc}}}{\delta \rho_{k}(\vec{r}) \delta \rho_{k^{\prime}}(\vec{r})} \frac{\partial \rho_{k}(\vec{r})}{\partial \kappa_{p q}}\right|_{0} \frac{\partial \rho_{k^{\prime}}(\vec{r})}{\partial B_{\alpha}}\right|_{0} \mathrm{~d} \vec{r} \\
& +\left.\int \frac{\delta E_{\mathrm{xc}}}{\delta \rho_{k}(\vec{r})} \frac{\partial^{2} \rho_{k}(\vec{r})}{\partial \kappa_{p q} \partial B_{\alpha}}\right|_{0} \mathrm{~d} \vec{r} .
\end{aligned}
$$

The first RHS term in Eq. (37) is an integral over the XC kernel

$$
\left.\left.\int \frac{\delta^{2} E_{\mathrm{xc}}}{\delta \rho_{k} \delta \rho_{k^{\prime}}} \frac{\partial \rho_{k}}{\partial \kappa_{a i}}\right|_{0} \frac{\partial \rho_{k^{\prime}}}{\partial B_{\alpha}}\right|_{0} \mathrm{~d} \vec{r}=-\int \frac{\delta^{2} E_{\mathrm{xc}}}{\delta \rho_{k} \delta \rho_{k^{\prime}}} \Omega_{i a ; k} \Omega_{j j ; k^{\prime}}^{B_{\alpha}} \mathrm{d} \vec{r},
$$

where $\Omega_{j j ; k^{\prime}}^{B_{\alpha}}$ can be expanded according to Eq. (29). Note that $\Omega_{j j ; k^{\prime}}^{B_{\alpha}}$ contains the direct term which only contributes at the SDFT level. The second RHS term in Eq. (37) is an integral over the $\mathrm{XC}$ potential magnetic derivative

$$
\left.\int \frac{\delta E_{\mathrm{xc}}}{\delta \rho_{k}} \frac{\partial^{2} \rho_{k}}{\partial \kappa_{a i} \partial B_{\alpha}}\right|_{0} \mathrm{~d} \vec{r}=-\int \frac{\delta E_{\mathrm{xc}}}{\delta \rho_{k}} \Omega_{i a ; k}^{B_{\alpha}} \mathrm{d} \vec{r} .
$$

Also here, $\Omega_{i a ; k}^{B_{\alpha}}$ is to be expanded according to Eq. (29).

Finally, we would like to present computationally useful expressions of LAO contributions to the XC part of the property gradient, Eqs. (38) and (39). For this purpose we will assume that the $\mathrm{XC}$ energy is a functional of the number and spin densities and their gradients (after Ref. 63):

$$
E_{\mathrm{xc}}=\int \varepsilon_{\mathrm{xc}}(n, s,(\nabla n \cdot \nabla n),(\nabla n \cdot \nabla s),(\nabla s \cdot \nabla s)) \mathrm{d} \vec{r} .
$$

We will only consider derivatives of the XC energy density which do not vanish when evaluated for a closed-shell reference system. Using the notation of this article $n=\rho_{0}$ and $s$ $=\sqrt{\rho_{\mu} \rho_{\mu}}$, the expression for the matrix elements that involve the XC potential, Eq. (39),

$$
\left.\int \frac{\delta E_{\mathrm{xc}}}{\delta \rho_{k}} \frac{\partial^{2} \rho_{k}}{\partial \kappa_{a i} \partial B_{\alpha}}\right|_{0} \mathrm{~d} \vec{r}=-\int \frac{\delta E_{\mathrm{xc}}}{\delta \rho_{k}} \Omega_{i a ; k}^{B_{\alpha}} \mathrm{d} \vec{r},
$$

can be evaluated using regular XC potential matrix distribution routines just by replacing the overlap distribution elements $\Omega_{i a ; k}$ by the field-differentiated LAO overlap distribution elements $\Omega_{i a ; k}^{B_{\alpha}}$. For molecular systems at closed-shell reference we can therefore use

$$
-\int \frac{\delta E_{\mathrm{xc}}}{\delta \rho_{k}} \Omega_{i a ; k}^{B_{\alpha}} \mathrm{d} \vec{r}=-\int\left[u_{0} \Omega_{i a ; 0}^{B_{\alpha}}+\vec{v}_{0} \cdot \nabla \Omega_{i a ; 0}^{B_{\alpha}}\right] \mathrm{d} \vec{r},
$$

where we employ the following scalar and vector overlap distribution pre-factors:

$$
\begin{gathered}
u_{0}=\left.\frac{\partial \varepsilon_{\mathrm{xc}}}{\partial n}\right|_{0}, \\
\vec{v}_{0}=\left.2 \frac{\partial \varepsilon_{\mathrm{xc}}}{\partial(\nabla n \cdot \nabla n)}\right|_{0} \nabla \rho_{0} .
\end{gathered}
$$

In practice only the first RHS term in Eq. (29) is explicitly integrated, the remaining terms are evaluated using the unperturbed KS matrix. Also the matrix elements that involve the XC kernel, Eq. (38), can be evaluated using a similar generic evaluation according to:

$$
-\int \frac{\delta^{2} E_{\mathrm{xc}}}{\delta \rho_{k} \delta \rho_{k^{\prime}}} \Omega_{i a ; k} \Omega_{j j ; k^{\prime}}^{B_{\alpha}} \mathrm{d} \vec{r}=-\int\left[u_{k}^{B_{\alpha}} \Omega_{i a ; k}+\vec{v}_{k}^{B_{\alpha}} \cdot \nabla \Omega_{i a ; k}\right] \mathrm{d} \vec{r},
$$

where $k$ now runs over $(0,1,2,3)$. Here we employ the following scalar and vector overlap distribution pre-factors $(\mu$ $=1,2,3)$ :

$$
u_{0}^{B_{\alpha}}=\left.\frac{\partial^{2} \varepsilon_{\mathrm{xc}}}{\partial n^{2}}\right|_{0} \rho_{0}^{B_{\alpha}}+\left.2 \frac{\partial^{2} \varepsilon_{\mathrm{xc}}}{\partial n \partial(\nabla n \cdot \nabla n)}\right|_{0}\left(\nabla \rho_{0} \cdot \nabla \rho_{0}^{B_{\alpha}}\right),
$$

$$
\begin{aligned}
& u_{\mu}^{B_{\alpha}}=\left.\frac{\partial^{2} \varepsilon_{\mathrm{xc}}}{\partial s^{2}}\right|_{0} \rho_{\mu}^{B_{\alpha}}+\left.\frac{\partial^{2} \varepsilon_{\mathrm{xc}}}{\partial s \partial(\nabla n \cdot \nabla s)}\right|_{0}\left(\nabla \rho_{0} \cdot \nabla \rho_{\mu}^{B_{\alpha}}\right), \\
& \vec{v}_{0}^{B_{\alpha}}=\left.2 \frac{\partial^{2} \varepsilon_{\mathrm{xc}}}{\partial n \partial(\nabla n \cdot \nabla n)}\right|_{0} \rho_{0}^{B_{\alpha}} \nabla \rho_{0}, \\
& +\left.4 \frac{\partial^{2} \varepsilon_{\mathrm{xc}}}{\partial(\nabla n \cdot \nabla n)^{2}}\right|_{0}\left(\nabla \rho_{0} \cdot \nabla \rho_{0}^{B_{\alpha}}\right) \nabla \rho_{0} \\
& +\left.2 \frac{\partial \varepsilon_{\mathrm{xc}}}{\partial(\nabla n \cdot \nabla n)}\right|_{0} \nabla \rho_{0}^{B_{\alpha}} \\
& \vec{v}_{\mu}^{B_{\alpha}}=\left.\frac{\partial^{2} \varepsilon_{\mathrm{xc}}}{\partial s \partial(\nabla n \cdot \nabla s)}\right|_{0} \rho_{\mu}^{B_{\alpha}} \nabla \rho_{0} \\
& +\left.\frac{\partial^{2} \varepsilon_{\mathrm{xc}}}{\partial(\nabla n \cdot \nabla s)^{2}}\right|_{0}\left(\nabla \rho_{0} \cdot \nabla \rho_{\mu}^{B_{\alpha}}\right) \nabla \rho_{0} \\
& +\left.2 \frac{\partial \varepsilon_{\mathrm{xc}}}{\partial(\nabla s \cdot \nabla s)}\right|_{0} \nabla \rho_{\mu}^{B_{\alpha}} .
\end{aligned}
$$


The derivatives of the density vector with respect to the $\alpha$ component of the magnetic field, $\rho_{0}^{B_{\alpha}}$ and $\rho_{\mu}^{B_{\alpha}}$, have been given in Eq. (26). There are two contributions to such derivatives: a direct contribution which involves the magnetic derivative of the London phase factor (first term in Eq. (29)) and the reorthonormalization contribution (second and third terms in Eq. (29)). In practice they are evaluated differently: the direct term is constructed explicitly by calculating $\frac{i}{2}\left(\vec{R}_{M N} \times \vec{r}\right)_{\alpha} \Omega_{\mu v ; k}$ in each grid point $(\vec{r})$ and contracting it with the density matrix $c_{\mu p}^{*} c_{\nu q}$. As already mentioned earlier, this term is zero for $\rho_{0}$. In order to evaluate the reorthonormalization contribution to $\rho_{0}^{B_{\alpha}}$ and $\rho_{\mu}^{B_{\alpha}}$, first the modified density matrix is constructed, $D_{\nu \mu}^{T}=c_{\mu p} T_{p q} c_{\nu q}^{\star}$ $+c_{p \mu}^{\star} T_{q p}^{\star} c_{q \nu}$, and then combined with the generalized overlap of atomic orbitals $\Omega_{\mu \nu ; k}=\chi_{\mu}^{\dagger} \boldsymbol{\Sigma}_{k} \chi_{\nu}$. We use an analogous algorithm to obtain the gradient of magnetic derivatives of density, $\nabla \rho_{0}^{B_{\alpha}}$ and $\nabla \rho_{\mu}^{B_{\alpha}}$. The reorthonormalization contributions to these quantities are easy to evaluate: the modified density matrix elements $D_{v \mu}^{T}$ are now multiplied with the elements of the generalized overlap gradient $\nabla \Omega_{\mu v ; k}$. The implementation of the direct contribution to $\nabla \rho_{0}^{B_{\alpha}}$ and $\nabla \rho_{\mu}^{B_{\alpha}}$ is slightly more elaborate, due to the fact that in each grid point the following term is evaluated:

$$
\begin{aligned}
\nabla\left[\frac{\mathrm{i}}{2}\left(\vec{R}_{M N} \times \vec{r}\right) \Omega_{\mu \nu ; k}\right]= & -\frac{\mathrm{i}}{2} \Omega_{\mu v ; k} R_{M N} \cdot(\nabla \times \vec{r}) \\
& +\frac{\mathrm{i}}{2}\left(\vec{R}_{M N} \times \vec{r}\right) c_{\mu p}^{*} c_{\nu q} \nabla \Omega_{\mu \nu ; k} .
\end{aligned}
$$

\section{COMPUTATIONAL DETAILS}

The extension of the four-component LAO HF code to accommodate $\mathrm{XC}$ contributions has been carried out using a locally modified development version of the DIRAC program. $^{45}$

All four-component relativistic calculations are based on the Dirac-Coulomb Hamiltonian. From the relevant twoelectron integral classes we have computed only (LL|LL) and (SS|LL) integrals explicitly (L stands for large and S stands for small component basis functions). At the SCF step the contribution of the rather expensive (SS|SS) integrals to the energy have been approximated by a classical Coulomb repulsion using tabulated small component charges according to Visscher, ${ }^{66}$ while we have simply omitted these integrals at the response step. In our experience, this is a very good approximation for the calculation of NMR shielding constants. ${ }^{27}$ We have also performed non-relativistic DFT calculations using the four-component Lévy-Leblond Hamiltonian ${ }^{57}$ in order to compare our implementation with the corresponding nonrelativistic implementation in Dalton. ${ }^{67}$ The results obtained using these two programs were found to be identical within numerical accuracy (numbers not shown).

Our test systems are the $\mathrm{HX}$ series $(\mathrm{X}=\mathrm{F}, \mathrm{Cl}, \mathrm{Br}$, I, $\mathrm{At})$, the $\mathrm{Xe}$ atom and the $\mathrm{Xe}$ dimer, the latter with two different internuclear distances: $4.0 \AA$ and $30.0 \AA$. The H-X distances have been taken from Ref. 68 and are $0.917 \AA$ (HF), $1.275 \AA$ ( $\mathrm{HCl}), 1.414 \AA(\mathrm{HBr})$, and $1.609 \AA$ (HI). For HAt we took the theoretical value of $1.737 \AA$, calculated at the
DC-CCSD level of theory by Styszynski ${ }^{69}$ We have employed Gaussian-type nuclear charge distributions using the recommended exponents. ${ }^{70}$

For HX compounds we have performed a series of calculations with different $\mathrm{XC}$ functionals: LDA (SVWNS), ${ }^{71,72}$ PP86 (which combines the correlation functional P86 (Ref. 73) with the exchange functional PW86, ${ }^{74}$ ) BP86, ${ }^{73,75}$ B3LYP, ${ }^{76} \mathrm{KT} 1$, and KT2. ${ }^{77}$ These are non-relativistic XC functionals, albeit evaluated using relativistic densities.

We have used three different schemes to represent the large-small component balance: RKB, UKB, and sMB. To represent the large component functions we have employed two different basis sets ( $T Z$ and $Q Z$ ). Basis sets denoted $T Z$ are aug-cc-pVTZ (Refs. 78 and 79) for H, F, and Cl, and Dyall's augmented core-valence triple-zeta basis ${ }^{80,81}$ for Br, I and At. Basis sets denoted $Q Z$ are aug-cc-pVQZ (Refs. 78 and 79) for $\mathrm{H}, \mathrm{F}$, and $\mathrm{Cl}$, and Dyall's augmented core-valence quadruplezeta basis ${ }^{81}$ for Br, I, and At.

For the Xe atom and the Xe dimer we have also performed DFT/SDFT calculations, but only using the KT2 functional (recommended by Keal and Tozer ${ }^{77}$ for calculations of the NMR shielding tensor), again with three different schemes for the kinetic balance. Two basis sets have been selected for the purpose: a triple zeta basis with additional diffuse and core correlating functions by Dyall (dyall.acv3z) ${ }^{80,81}$ and a basis set optimized for NMR shieldings by Vaara and Pyykkö ${ }^{82}$ and extended by a diffuse $s$-exponent. ${ }^{7}$ The large component bases contain 29s22p14d4f1g and 25s19p22d11f8g function ranges, respectively, and we will refer to these basis sets as Dyall and Vaara.

Throughout all calculations a tight convergence threshold has been imposed: $10^{-7}$ for the electronic gradient in SCF calculations and $10^{-9}$ for the norm of the residual vector relative to the property gradient in the linear response calculations.

\section{RESULTS AND DISCUSSION}

\section{A. Performance of different functionals and comparison with literature}

\section{HX compounds}

We begin this section with a discussion of isotropic shielding constants of the hydrogen halides calculated with different XC functionals, the DC Hamiltonian, the sMB scheme, and using the $Q Z$ basis set. The results of these calculations are presented in Table I, where they can be compared with the results obtained by Komorovský et al. ${ }^{30,38}$ and with available experimental values. In addition, we have performed corresponding calculations using UKB and obtain results identical to the sMB values (matching within $0.01 \mathrm{ppm}$ except At in HAt, where these results agree to $0.1 \mathrm{ppm}$ ). This confirms that the sMB scheme proposed in this article describes well the magnetic balance, Eq. (11), while remaining computationally economic and straightforward to implement.

In order to compare our magnetic balance scheme with explicit RMB, we have performed sMB-SDFT/BP86 calculations which can be compared with the uncoupled mDKSRMB-GIAO/BP86 results of Ref. 30. We observe that the calculated isotropic shielding values by Komorovský et al. 
TABLE I. Isotropic shielding values (in ppm) of $\mathrm{HX}(\mathrm{X}=\mathrm{F}, \mathrm{Cl}, \mathrm{Br}, \mathrm{I}, \mathrm{At})$ calculated with the Dirac-Coulomb Hamiltonian, sMB balance, and LAOs (unless otherwise stated) and the $Q Z$ basis set.

\begin{tabular}{|c|c|c|c|c|c|c|c|c|c|c|c|c|c|c|c|c|c|}
\hline & & \multicolumn{2}{|c|}{ LDA } & \multicolumn{2}{|c|}{ B3LYP } & \multicolumn{2}{|c|}{ KT1 } & \multicolumn{2}{|c|}{ KT2 } & \multicolumn{2}{|c|}{ PP86 } & \multicolumn{2}{|c|}{ BP86 } & \multicolumn{2}{|c|}{$\mathrm{PP}^{\mathrm{a}} \mathrm{a}^{\mathrm{a}}$} & \multirow{2}{*}{$\frac{\mathrm{BP}^{\mathrm{b}} \mathrm{b}^{\mathrm{f}}}{\mathrm{SDFT}^{\mathrm{c}}}$} & \multirow[b]{2}{*}{ Experiment } \\
\hline & & DFT & SDFT & DFT & SDFT & DFT & SDFT & DFT & SDFT & DFT & SDFT & DFT & SDFT & DFT & SDFT & & \\
\hline \multirow[t]{2}{*}{$\mathrm{HF}$} & $\mathrm{H}$ & 29.08 & 29.10 & 29.34 & 29.37 & 30.09 & 30.13 & 29.82 & 29.87 & 29.68 & 29.72 & 29.77 & 29.81 & 29.20 & 29.24 & 30.5 & $28.72^{\mathrm{d}}, 28.50^{\mathrm{e}}, 28.5 \pm 0.2^{\mathrm{f}}$ \\
\hline & $\mathrm{F}$ & 418.82 & 419.00 & 414.96 & 415.14 & 414.85 & 415.16 & 415.34 & 415.69 & 414.50 & 414.73 & 414.52 & 414.77 & 411.1 & 411.4 & 416.2 & $410 \pm 6^{\mathrm{g}}$ \\
\hline \multirow[t]{2}{*}{$\mathrm{HCl}$} & $\mathrm{H}$ & 31.09 & 31.25 & 31.72 & 31.95 & 31.78 & 32.00 & 31.68 & 31.98 & 31.84 & 32.12 & 31.79 & 32.03 & 31.63 & 31.92 & 32.6 & $31.06^{\mathrm{e}}$ \\
\hline & $\mathrm{Cl}$ & 981.57 & 982.39 & 967.25 & 968.18 & 987.11 & 988.38 & 984.76 & 986.22 & 966.63 & 967.93 & 970.78 & 972.00 & 935.0 & 936.3 & 973.9 & $952^{\mathrm{h}}$ \\
\hline \multirow[t]{2}{*}{$\mathrm{HBr}$} & $\mathrm{H}$ & 33.75 & 34.73 & 35.02 & 36.42 & 34.60 & 35.95 & 34.63 & 36.44 & 35.03 & 36.65 & 34.73 & 36.16 & 33.52 & 35.13 & 36.4 & $34.96^{\mathrm{e}}$ \\
\hline & $\mathrm{Br}$ & 2901.51 & 2908.83 & 2869.87 & 2878.58 & 2914.44 & 2923.95 & 2904.77 & 2916.72 & 2860.89 & 2873.82 & 2870.34 & 2881.92 & 2876.0 & 2887.9 & 2899.4 & $2617^{\mathrm{h}}$ \\
\hline \multirow[t]{2}{*}{ HI } & $\mathrm{H}$ & 39.19 & 42.20 & 41.79 & 46.04 & 40.17 & 44.28 & 40.42 & 45.96 & 41.09 & 45.71 & 40.61 & 44.72 & 39.34 & 43.82 & 46.3 & $43.86^{\mathrm{e}}$ \\
\hline & I & 5705.32 & 5738.77 & 5648.45 & 5688.14 & 5732.26 & 5772.58 & 5707.05 & 5760.82 & 5631.30 & 5686.76 & 5639.34 & 5691.67 & 5661.6 & 5705.1 & 5716.3 & $4510^{\mathrm{h}}$ \\
\hline \multirow[t]{2}{*}{ HAt } & $\mathrm{H}$ & 50.07 & 57.52 & 55.79 & 66.24 & 52.13 & 62.68 & 52.61 & 66.80 & 53.46 & 63.81 & 52.49 & 61.66 & & & & \\
\hline & At & 16290.46 & 16613.22 & 16401.59 & 16765.53 & 16346.85 & 16745.68 & 16282.02 & 16818.86 & 16123.69 & 16591.04 & 16133.59 & 16596.95 & & & & \\
\hline
\end{tabular}

aef. 38, no LAOs, DFT/SDFT denoted uncoupled/coupled.

${ }^{\mathrm{b}}$ Reference 30 .

${ }^{c} \mathrm{mDKS}-\mathrm{RMB}$ scheme with LAOs.

${ }^{\mathrm{d}}$ Ref. 91 , absolute experimental shielding.

${ }^{\mathrm{e}}$ Ref. 90, gas-phase NMR measurement; the rovibrational contribution at $\mathrm{T}=300 \mathrm{~K}$ was estimated at $20.52 \mathrm{ppm} .{ }^{95}$

${ }^{\mathrm{f}}$ Ref. 96, the value is a combination of theoretically calculated diamagnetic shielding and experimental spin-rotation constant; the rovibrational corrections were estimated at $210.4 \mathrm{ppm}($ Ref. 97$)$ or $210.8 \mathrm{ppm}(\mathrm{Ref}$. 95 ) at $\mathrm{T}=300 \mathrm{~K}$.

${ }^{g}$ Ref. 89, gas-phase NMR chemical shifts with respect to $\mathrm{CH}_{4}$ converted into absolute shieldings using. $\sigma_{\mathrm{H}}\left(\mathrm{CH}_{4}\right)=30.61$ ppm from Ref. 90.

${ }^{\mathrm{h}}$ The value is a combination of experimental spin-rotation constant and calculated diamagnetic shielding within the atom dipole model proposed in Ref. 98. 
are systematically larger than ours. This difference can in part be attributed to differences in basis sets: in Ref. 30 the Fægri basis sets (augmented by additional $s-, p$-, and $d$-functions) for $\mathrm{Br}$ and I and the IGLO-III basis set for $\mathrm{H}, \mathrm{F}$, and $\mathrm{Cl}$ have been used, while our results have been obtained using the $Q Z$ basis sets detailed further above. For the HI molecule we have therefore repeated the sMB-SDFT/BP86 calculations using the same geometry and basis set as in Ref. 30 and obtain $44.8 \mathrm{ppm}$ for $\sigma(\mathrm{H})$ and $5668 \mathrm{ppm}$ for $\sigma(\mathrm{I})$, which can be compared with $46.3 \mathrm{ppm}$ for $\sigma(\mathrm{H})$ and $5716 \mathrm{ppm}$ for $\sigma(\mathrm{I})$ obtained with the methodology discussed in Ref. 30. However, a detailed comparison between the DIRAC and ReSpect codes revealed that the calculations differ already in the SCF energies (numbers not shown) with the consequence that the shielding constants (energy derivatives) differ as well. Due to density fitting and a numerical scheme employed in ReSpect, the mDKS-RMB-GIAO implementation in this code therefore cannot be directly compared with our fully analytical sMB-SDFT implementation.

In addition to BP86, we report results for four other XC functionals: B3LYP, KT1, KT2, and PP86. The relative differences in isotropic shielding constants between different GGA functionals are small with differences smaller than $2 \%$ (for both DFT and SDFT). The results obtained with LDA are close to the GGA values; for instance, for iodine in HI molecule the DFT/LDA isotropic shielding constant equals $5705 \mathrm{ppm}$ while the DFT/GGA values vary from $5631 \mathrm{ppm}$ (PP86) to $5732 \mathrm{ppm}$ (KT1). There is therefore no substantial effect of adding the gradient corrections for the systems under study. The same trend is observed for SDFT.

Extensive studies on the NMR shielding tensor in nonrelativistic formalism showed that in many cases XC approximations fail to reproduce accurate values of this property and the choice of functional strongly depends on the system under study. ${ }^{83-85}$ It has been shown that the values of NMR isotropic shielding constant calculated with KealTozer functionals KT1 $1{ }^{77} \mathrm{KT} 2,{ }^{77}$ and KT $3{ }^{86}$ are often improved over those obtained with other functionals. ${ }^{77,86,87}$ These functionals are specially designed for the calculation of NMR shielding constants and most notably reduce the HOMO-LUMO gap, which in the non-relativistic formalism improves the paramagnetic part of the NMR shielding tensor. In relativistic formalism the performance of Keal-Tozer functionals is not fully tested. To our knowledge, only one study of the performance of different functionals in relativistic formalism has been reported in the literature. ${ }^{88}$ Armangué et al. have used the ADF program, which employs the twocomponent zero-order regular approximation (ZORA) Hamiltonian (however, this has not been specified in Ref. 88) for determining the NMR shielding tensor for a wide range of molecules. The KT2 functional predicted these values very well.

In our study we observe that there are no significant differences between values obtained with KT1/KT2 and other functionals. However, it is worth to note that KT1 isotropic shielding constants for heavy atoms ( $\mathrm{X}$ in $\mathrm{HX}$ ) are the largest of all obtained values. Unfortunately, the lack of reliable experimental results does not allow us to assess the performance of the different functionals.
Turning now to the proton shielding constants, our results are generally in rather good agreement with experimental values for the proton isotropic shielding in the HX series. ${ }^{89}$ However, it should be taken into account that these values have been recalculated from the experimental chemical shift values reported in Ref. 89 (in 1958) using the $\mathrm{H}$ shielding in $\mathrm{CH}_{4}$ (reported in 1978 in Ref. 90). Therefore, the reference value can be burdened by uncertainties connected with the conversion of the relative chemical shielding to the absolute shielding constants. The most reliable experimental value is the absolute isotropic shielding available for $\mathrm{H}$ in $\mathrm{HF}$ (28.72 ppm (Ref. 91)). Our results, as well as values presented by Komorovský et al. ${ }^{30}$ overestimate this experimental value by about $5 \%$. For the heavy nuclei, the experimental values of the spin-rotation constants have been used to determine the paramagnetic part of the shielding tensor, which has been combined with calculated diamagnetic values. However, it has been demonstrated that this relation is not valid for heavy systems, ${ }^{43}$ therefore the values of the isotropic shielding are not accurate enough..$^{92}$ For instance for I, such predicted value is $4510 \mathrm{ppm}$ which is significantly smaller than the values presented here: $5761 \mathrm{ppm}$ and $5692 \mathrm{ppm}$ (sMB-SDFT/KT2 and sMB-SDFT/BP86, respectively). This is also the case for the value of 5716 ppm obtained in Ref. 30 (mDKS-RMBGIAO/BP86).

\section{Xe atom}

For the Xe atom we have selected the results obtained by means of the SDFT method with the sMB scheme and using the Vaara basis set as representative results (Table III). The Vaara basis set was optimized for Xe NMR shielding constants and is substantially larger than the Dyall basis set. We have selected the KT2 functional for the study of the Xe atom and our prediction for the isotropic shielding constant of $\mathrm{Xe}$ is $7045 \mathrm{ppm}$ (Table III).

The available theoretical literature values for this constant were compared in Ref. 46 for a wide range of methods and Hamiltonians: non-relativistic coupled-cluster method with single, double, and perturbative triple excitations $(\operatorname{CCSD}(\mathrm{T}))$, non-relativistic Møller-Plesset perturbation theory to second order (MP2), Breit-Pauli perturbation theory (BPPT) based on a HF and KS reference (where relativistic effects are calculated in a perturbative manner), as well as four-component relativistic HF. Results obtained by relativistic methods in Ref. 46 (with Dyall basis set of QZ quality), $7020 \mathrm{ppm}$ (4c rel. HF) and $6747 \mathrm{ppm}$ (HF BPPT), are comparable with our sMB-SDFT/KT2 value of $7029 \mathrm{ppm}$, obtained using TZ-quality Dyall basis set. In Ref. 46, the authors have demonstrated that electron correlation effects on the Xe shielding tensor calculated within non-relativistic formalism are small and so are cross-couplings effects between electron correlation and relativity. We can confirm this by our DFT/SDFT predictions: when compared with fourcomponent relativistic HF results taken from Ref. 46, the difference of $10 \mathrm{ppm}$ constitutes only $0.6 \%$ of the HF value and should probably be attributed to basis set differences. We will return to a detailed discussion of basis set differences in Sec. IV C. Before that we would like to mention that the 
TABLE II. Isotropic shielding values (in ppm) of $\mathrm{HX}(\mathrm{X}=\mathrm{F}, \mathrm{Cl}, \mathrm{Br}, \mathrm{I}, \mathrm{At})$ calculated using the DC Hamiltonian, $\mathrm{KT} 2$ functional, LAOs and various balance schemes (RKB, UKB, sMB) and basis sets.

\begin{tabular}{|c|c|c|c|c|c|c|c|c|}
\hline & & \multirow[b]{2}{*}{ Basis } & \multicolumn{3}{|c|}{ DFT } & \multicolumn{3}{|c|}{ SDFT } \\
\hline & & & RKB & UKB & sMB & RKB & UKB & sMB \\
\hline \multirow[t]{4}{*}{$\mathrm{HF}$} & \multirow[t]{2}{*}{$\mathrm{H}$} & $T Z$ & 28.80 & 29.95 & 29.95 & 28.85 & 30.00 & 30.00 \\
\hline & & $Q Z$ & 29.43 & 29.82 & 29.82 & 29.48 & 29.87 & 29.87 \\
\hline & \multirow[t]{2}{*}{$\mathrm{F}$} & $T Z$ & 323.14 & 415.71 & 415.71 & 323.48 & 416.05 & 416.05 \\
\hline & & $Q Z$ & 343.55 & 415.34 & 415.34 & 343.90 & 415.69 & 415.69 \\
\hline \multirow[t]{4}{*}{$\mathrm{HCl}$} & \multirow{2}{*}{$\mathrm{H}$} & $T Z$ & 30.86 & 31.73 & 31.73 & 31.14 & 32.01 & 32.01 \\
\hline & & $Q Z$ & 31.39 & 31.68 & 31.68 & 31.68 & 31.98 & 31.98 \\
\hline & \multirow[t]{2}{*}{$\mathrm{Cl}$} & $T Z$ & 695.14 & 986.68 & 986.68 & 696.54 & 988.12 & 988.12 \\
\hline & & $Q Z$ & 707.03 & 984.76 & 984.76 & 708.45 & 986.22 & 986.22 \\
\hline \multirow[t]{4}{*}{$\mathrm{HBr}$} & \multirow[t]{2}{*}{$\mathrm{H}$} & $T Z$ & 33.70 & 34.50 & 34.50 & 35.43 & 36.22 & 36.22 \\
\hline & & $Q Z$ & 34.38 & 34.63 & 34.63 & 36.19 & 36.44 & 36.44 \\
\hline & \multirow[t]{2}{*}{$\mathrm{Br}$} & $T Z$ & 2747.91 & 2901.80 & 2901.80 & 2759.62 & 2913.54 & 2913.54 \\
\hline & & $Q Z$ & 2820.59 & 2904.77 & 2904.77 & 2832.53 & 2916.72 & 2916.72 \\
\hline \multirow[t]{4}{*}{$\mathrm{HI}$} & \multirow[t]{2}{*}{$\mathrm{H}$} & $T Z$ & 39.26 & 40.05 & 40.05 & 44.54 & 45.33 & 45.33 \\
\hline & & $Q Z$ & 40.14 & 40.42 & 40.42 & 45.68 & 45.96 & 45.96 \\
\hline & \multirow[t]{2}{*}{ I } & $T Z$ & 5255.85 & 5702.63 & 5702.63 & 5308.58 & 5755.50 & 5755.50 \\
\hline & & $Q Z$ & 5309.75 & 5707.06 & 5707.05 & 5363.44 & 5760.83 & 5760.82 \\
\hline \multirow[t]{4}{*}{ HAt } & \multirow[t]{2}{*}{$\mathrm{H}$} & $T Z$ & 50.90 & 51.78 & 51.78 & 64.41 & 65.29 & 65.29 \\
\hline & & $Q Z$ & 52.30 & 52.61 & 52.61 & 66.48 & 66.80 & 66.80 \\
\hline & \multirow[t]{2}{*}{ At } & $T Z$ & 15846.74 & 16261.13 & 16261.29 & 16377.52 & 16792.59 & 16792.82 \\
\hline & & $Q Z$ & 15893.76 & 16282.02 & 16282.02 & 16430.21 & 16818.83 & 16818.86 \\
\hline
\end{tabular}

calculation of the isotropic shielding constant of Xe has also been reported in Ref. 30 using the mDKS-RMB-GIAO scheme and the BP86 functional, where the authors have used Dyall's TZ basis set and a numerical scheme for evaluating the GGA kernel matrix elements. Within this numerical scheme they have reported the value $7033 \mathrm{ppm}$. The sMBSDFT/KT2 approach with Dyall basis set obtained in this work yields $7029 \mathrm{ppm}$, therefore, for this system, these two four-component implementations agree well, despite using different functionals.

\section{B. DFT vs. SDFT}

For all calculated shielding constants we present DFT and SDFT results, where the latter include non-collinear spin magnetization contributions. Inclusion of spin magnetization leads to an increase of the isotropic shielding constants for all systems under study (Table I). However, for heavy nuclei this difference is not substantial: for instance the SDFT values are only $0.2 \%$ larger for Xe, $0.9 \%$ for $\mathrm{I}(\mathrm{HI})$, and $3 \%$ for At (HAt). The effect of spin magnetization is significant for the $\mathrm{H}$ atom (HX series) and in the heaviest systems it can be as large as $27 \%$ (HAt with KT2 functional).

The differences between the DFT and SDFT approaches can be compared with the results obtained with mDKS-RMBCGO/PP86 in Ref. 38, where the uncoupled scheme involves only charge density (in analogy with our DFT results) and the coupled scheme additionally includes spin magnetization (in analogy with our SDFT results). Although Ref. 38 employed a CGO approach, the relative differences between the uncoupled and coupled schemes are comparable with the relative differences between DFT and SDFT methods presented here.
As an example, our calculations for HI (PP86 functional) lead to SDFT values which are larger by $11.2 \%$ for $\mathrm{H}$ and $1.0 \%$ for I compared to the corresponding DFT results. The authors of Ref. 38 have obtained similar differences: $11.4 \%$ and $0.8 \%$ for $\mathrm{H}$ and I, respectively.

\section{Basis set checks and balances}

In Tables II-IV we compare the performance of RKB, UKB, and sMB schemes in the calculations of NMR shielding constants. In all cases UKB and SMB calculations yield almost identical results and small differences only occur for the heaviest atoms. For instance, the Xe atom shielding constants calculated with UKB-SDFT/KT2 and SMB-SDFT/KT2 are $7045 \mathrm{ppm}$ and $7045 \mathrm{ppm}$, respectively (Vaara basis set). For the HX series these differences are only on the first decimal digit, even for the heaviest atoms, I and At. This behavior is as expected since the set of small-component basis functions in the sMB scheme is constructed from the RKB balance

TABLE III. The influence of kinetic balance (RKB, UKB, sMB) and basis set (Dyall, Vaara) on the value of the isotropic shielding constant (in ppm) in Xe evaluated using the DC Hamiltonian and the KT2 functional with LAO.

\begin{tabular}{lccccc}
\hline \hline & Basis & RMB & RKB & UKB & sMB \\
\hline DFT & Dyall & & 6558.38 & 7015.10 & 7015.10 \\
& Vaara & & 7032.50 & 7030.55 & 7030.88 \\
\multirow{3}{*}{ SDFT } & Dyall & & 6571.90 & 7028.79 & 7028.78 \\
& Vaara & & 7046.74 & 7044.82 & 7045.11 \\
& Ref. 30 & 7033.3 & & & \\
\hline \hline
\end{tabular}

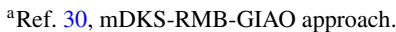


TABLE IV. The influence of kinetic balance (RKB, UKB, sMB) and basis set (Dyall, Vaara) on the value of isotropic shielding constants (in ppm) in the Xe dimer evaluated using the DC Hamiltonian and the KT2 functional.

\begin{tabular}{|c|c|c|c|c|c|c|c|c|c|}
\hline & & \multirow[b]{2}{*}{ Basis } & & \multicolumn{3}{|c|}{$R(\mathrm{Xe}-\mathrm{Xe})=4 \AA$} & \multicolumn{3}{|c|}{$R(\mathrm{Xe}-\mathrm{Xe})=30 \AA$} \\
\hline & & & & RKB & UKB & sMB & RKB & UKB & sMB \\
\hline \multirow[t]{4}{*}{ LAO } & DFT & Dyall & & 6459.45 & 6915.99 & 6915.98 & 6558.38 & 7015.10 & 7015.10 \\
\hline & & Vaara & & 6932.89 & 6930.96 & 6931.33 & 7032.49 & 7030.55 & 7033.88 \\
\hline & SDFT & Dyall & & 6472.78 & 6929.49 & 6929.47 & 6571.90 & 7028.78 & 7028.78 \\
\hline & & Vaara & & 6944.25 & 6942.38 & 6942.68 & 7046.73 & 7044.82 & 7045.11 \\
\hline \multirow[t]{8}{*}{ CGO at $\mathrm{Xe}(1)$} & DFT & Dyall & $\mathrm{Xe}(1)$ & 6476.33 & 6941.18 & 6941.17 & 6560.82 & 7018.68 & 7018.68 \\
\hline & & & $\mathrm{Xe}(2)$ & 6458.37 & 6915.17 & 6915.16 & 6558.38 & 7015.10 & 7015.10 \\
\hline & & Vaara & $\mathrm{Xe}(1)$ & 6933.13 & 6931.27 & 6931.59 & 7032.53 & 7030.60 & 7030.93 \\
\hline & & & $\mathrm{Xe}(2)$ & 6934.87 & 6932.96 & 6933.29 & 7032.50 & 7030.55 & 7030.88 \\
\hline & SDFT & Dyall & $\mathrm{Xe}(1)$ & 6489.15 & 6954.16 & 6954.15 & 6574.33 & 7032.36 & 7032.36 \\
\hline & & & $\mathrm{Xe}(2)$ & 6471.15 & 6928.12 & 6928.10 & 6571.90 & 7028.78 & 7028.79 \\
\hline & & Vaara & $\mathrm{Xe}(1)$ & 6946.55 & 6944.73 & 6945.01 & 7046.77 & 7044.87 & 7045.16 \\
\hline & & & $\mathrm{Xe}(2)$ & 6948.23 & 6946.36 & 6946.64 & 7046.73 & 7044.83 & 7045.12 \\
\hline
\end{tabular}

condition extended by the UKB complement (as explained in Sec. II A).

Within the sMB scheme the differences between the results obtained for two basis sets, $T Z$ and $Q Z$ for HX or Dyall and Vaara for Xe atom and Xe dimer, are much smaller than using RKB. Therefore, the sMB scheme speeds up the basis set convergence and enables to get better results with compact basis sets. For example, using RKB the difference for the Xe shielding constant between the Dyall and the Vaara basis set is $7.2 \%$, whereas the difference is only $0.2 \%$ using sMB. This comparison has already been discussed in Ref. 27: the RKB approach does not generate all small component basis functions needed to saturate the small component space in the presence of perturbation originating from external magnetic field and to assure that the magnetic balance is preserved.

Another conclusion regarding the choice of basis sets which can be drawn from our study is that RKB and sMB results using the Vaara basis set for the Xe atom and Xe dimer are very similar. Therefore, these basis set can be regarded as saturated. For HX compounds there is no significant improvement with a change of basis set from $T Z$ to $Q Z$, however, for both basis sets there is a large difference between RKB and sMB results. For instance, the differences for the I shielding constant is $8.4 \%(T Z)$ or $7.4 \%(Q Z)$.

\section{LAO vs. CGO}

Finally, we would like to demonstrate the importance of using LAOs in four-component calculations of NMR shielding tensor. For this purpose, we have performed CGO calculations for $\mathrm{Xe}_{2}$, in which the gauge origin has been placed on one of the two nuclei. The calculations have been carried out for two inter-atomic distances: $R(\mathrm{Xe}-\mathrm{Xe})=4 \AA$ and $R(\mathrm{Xe}-$ $\mathrm{Xe})=30 \AA$ A . We have employed two different basis sets (Dyall and Vaara) and again three balance schemes (RKB, UKB, and sMB).

The results obtained using the KT2 functional are collected in Table IV. We observe that for the Xe dimer with $R(\mathrm{Xe}-\mathrm{Xe})=30 \AA$ the shielding constant for the nucleus placed at the CGO is further away from the LAO value than for the CGO-distant nucleus. The observation that the nucleus distant from the CGO is better described is at first counterintuitive, but is correct and has been explained recently by Malkina et al. ${ }^{93}$ where the shielding tensor of a nucleus in a system of two non-interacting nuclei was analyzed with the conclusion that the dominating contribution to the shielding tensor (the diamagnetic part) has an inverse dependence on the distance between the nucleus and the gauge origin.

We also notice that $\mathrm{LAO}$ results for $R(\mathrm{Xe}-\mathrm{Xe})=30$ $\AA$ are identical to the atomic results. This observation additionally supports the correctness of our implementation because we can approximately regard this system as two non-interacting nuclei.

Predictably, there is an important basis set effect when comparing the Vaara basis set with the Dyall basis set. The former has been designed for calculations of the Xe NMR shielding tensor and is significantly larger than the Dyall basis set. As expected, the CGO values are closer to the LAO results for the Vaara basis. For $R(\mathrm{Xe}-\mathrm{Xe})=30 \AA$ the two atoms have almost identical shielding constants.

\section{CONCLUSIONS}

We have presented an extension of linear response theory in perturbation-dependent basis to four-component relativistic DFT and SDFT. We have confirmed the conclusions from Ref. 27 about the slow convergence of results when RKB scheme is used: in order to saturate the space of small-component functions, extensive basis sets for the large component have to be used. Instead, the simple scheme for magnetic balance (sMB) has proved to be a useful and computationally economic way to calculate the NMR shielding tensor yielding results comparable with the mDKSRMB-GIAO method. ${ }^{30}$ The main advantages of the present formulation are the fully analytical implementation and the possibility to use hybrid functionals. The presented formulas are general and make it possible to extend the formulation to meta-GGA or hyper-GGA functional approximations or current density functionals. Moreover, only a small effort 
would be required to extend the implementation to other magnetic properties, including magnetizabilities.

The correctness of the presented formulation has been verified by calculations on simple test systems including HX $(\mathrm{X}=\mathrm{F}, \mathrm{Cl}, \mathrm{Br}, \mathrm{I}, \mathrm{At})$, the $\mathrm{Xe}$ atom, and the Xe dimer. In all cases we have achieved a close agreement with results obtained by a similar four-component DFT implementation ${ }^{30,38}$ but differences persist due to density fitting and the numerical integral scheme employed in the latter code. Interestingly, the contributions from spin magnetization, entering the molecular Hessian and the property gradient, are negligible for the shielding of heavy nuclei, but significant for hydrogen atoms in heavy HX molecules (10\% in HI). The formulation presented here has already been used to determine the shielding tensor in $\mathrm{XY}$ molecules, where $\mathrm{X}, \mathrm{Y}=\mathrm{N}, \mathrm{P}, \mathrm{As}^{92}$ and for studies of the $\mathrm{PH}_{3}$ molecule, where calculations were further used to redefine the absolute shielding scale for ${ }^{31} \mathrm{P} .{ }^{94}$

To sum up, the implementation has been well tested for small molecules, and is now ready for production calculations of the NMR shielding tensors in larger compounds of chemical interest containing heavy atoms. The next step would be to add the vibrational corrections and to account for solvent effects within our four-component scheme. We hope that the presented implementation, to be included in the freely distributed DIRAC code, will contribute to a more widespread use of relativistic calculations of NMR properties as a tool complementing the NMR experiment.

\section{ACKNOWLEDGMENTS}

The authors are grateful to M. Repiský and S. Komorovský for providing the basis set for $\mathrm{HI}$ in order to compare two working implementations. We acknowledge a grant of computer time from the Norwegian Supercomputing Program (Notur). M.O. was supported by Foundation for Polish Science (Project operated within the Foundation for Polish Science MPD Programme co-financed by the EU European Regional Development Fund) and by Ph.D. grant number N N204 116539. R.B. kindly acknowledges support from the Norwegian Research Council through a Centre of Excellence Grant (Grant No. 179568/V30).

${ }^{1}$ M. Kaupp, M. Buhl, and V. G. Malkin, Calculation of NMR and EPR Parameters: Theory and Applications (Wiley-VCH, Berlin, 2004).

${ }^{2}$ N. F. Ramsey, Phys. Rev. 77(4), 567 (1950).

${ }^{3}$ N. F. Ramsey, Phys. Rev. 78(6), 699 (1950).

${ }^{4}$ N. F. Ramsey, Phys. Rev. 83(3), 540 (1951).

${ }^{5}$ N. F. Ramsey, Phys. Rev. 86(2), 243 (1952).

${ }^{6}$ P. Pyykkö, Theor. Chem. Acc. 103, 214 (2000).

${ }^{7}$ T. Saue, Spin-interactions and the non-relativistic of electrodynamics, Advances in Quantum Chemistry Vol. 48 (Academic Press, New York, 2005), pp. 383-405.

${ }^{8}$ M. A. M. Forgeron, M. Gee, and R. E. Wasylishen, J. Phys. Chem. A. 108(22), 4895 (2004).

${ }^{9}$ P. Manninen, K. Ruud, P. Lantto, and J. Vaara, J. Chem. Phys. 122(11), 114107 (2005); 124, 149901 (2006).

${ }^{10}$ S. K. Wolff, T. Ziegler, E. van Lenthe, and E. J. Baerends, J. Chem. Phys. 110(16), 7689 (1999).

${ }^{11}$ P. Pyykkö, A. Görling, and N. Rösch, Mol. Phys. 61(1), 195 (1987).

${ }^{12}$ M. Kaupp, O. L. Malkina, V. G. Malkin, and P. Pyykkö, Chem. Eur. J. 4(1), 118 (1998)

${ }^{13}$ C. J. Jameson and A. C. de Dios, Theoretical and Physical Aspects of Nuclear Shielding, Nuclear Magnetic Resonance Vol. 36 (The Royal Society of Chemistry, London, 2007), pp. 50-71.
${ }^{14}$ L. B. Casabianca and A. C. de Dios, J. Chem. Phys. 128(5), 052201 (2008).

${ }^{15}$ J. Vaara, Phys. Chem. Chem. Phys. 9(40), 5399 (2007).

${ }^{16}$ L. Cheng, Y. Xiao, and W. Liu, J. Chem. Phys. 130(14), 144102 (2009); 131, 019902 (2009).

${ }^{17}$ H. F. Hameka, Rev. Mod. Phys. 34, 87 (1962).

${ }^{18}$ S. T. Epstein, The Variational Method in Quantum Chemistry (Academic Press, New York, 1974).

${ }^{19}$ W. Kutzelnigg, J. Mol. Struct.: THEOCHEM 202, 11 (1989).

${ }^{20} \mathrm{C}$. van Wüllen, Chemical shifts with Hartree-Fock and density-functional methods, in Calculation of NMR and EPR Parameters, edited by M. Kaupp, M. Bühl and V. G. Malkin (Wiley-VCH, Weinheim, 2004).

${ }^{21}$ F. London, J. Phys. Radium 8(10), 397 (1937).

${ }^{22}$ R. Ditchfield, Mol. Phys. 27(4), 789 (1974).

${ }^{23}$ A. E. Hansen and T. D. Bouman, J. Chem. Phys. 82(11), 5035 (1985).

${ }^{24}$ A. C. de Dios, Prog. Nucl. Magn. Reson. Spectrosc. 29(3-4), 229 (1996).

${ }^{25}$ H. M. Quiney, H. Skaane, and I. P. Grant, Ab Initio Relativistic Quantum Chemistry: Four-Components Good, Two-Components Bad!, Advances in Quantum Chemistry Vol. 36 (Academic Press, New York, 1999), pp. 1-49.

${ }^{26}$ S. Hamaya, H. Maeda, M. Funaki, and H. Fukui, J. Chem. Phys. 129(22), 224103 (2008).

${ }^{27}$ M. Iliaš, T. Saue, T. Enevoldsen, and H. J. A. Jensen, J. Chem. Phys. 131(12), 124119 (2009).

${ }^{28}$ S. Hamaya and H. Fukui, Bull. Chem. Soc. Jpn. 83(6), 635 (2010).

${ }^{29}$ L. Cheng, Y. Xiao, and W. Liu, J. Chem. Phys. 131(24), 244113 (2009).

${ }^{30}$ S. Komorovský, M. Repiský, O. L. Malkina, and V. G. Malkin, J. Chem. Phys. 132(15), 154101 (2010).

${ }^{31}$ G. A. Aucar, T. Saue, L. Visscher and H. J. A. Jensen, J. Chem. Phys. 110(13), 6208 (1999).

${ }^{32}$ W. Kutzelnigg, J. Comput. Chem. 20(12), 1199 (1999).

${ }^{33}$ W. Kutzelnigg, Phys. Rev. A. 67(3), 032109 (2003).

${ }^{34}$ M. M. Sternheim, Phys. Rev. 128, 676 (1962).

${ }^{35}$ P. Pyykkö, Chem. Phys. 74(1), 1 (1983).

${ }^{36}$ Y. Xiao, W. Liu, L. Cheng, and D. Peng, J. Chem. Phys. 126(21), 214101 (2007).

${ }^{37}$ L. Visscher, Magnetic Balance and Explicit Diamagnetic Expressions for Nuclear Magnetic Resonance Shielding Tensors, Advances in Quantum Chemistry Vol. 48 (Academic Press, New York, 2005), pp. 369-381.

${ }^{38}$ S. Komorovský, M. Repiský, O. L. Malkina, V. G. Malkin, I. M. Ondík, and M. Kaupp, J. Chem. Phys. 128(10), 104101 (2008).

${ }^{39}$ Y. Ishikawa, T. Nakajima, M. Hada, and H. Nakatsuji, Chem. Phys. Lett. 283(1-2), 119 (1998).

${ }^{40}$ M. Hada, Chem. Phys. Lett. 310(3-4), 342 (1999).

${ }^{41}$ M. Hada, Chem. Phys. Lett. 321(5-6), 452 (2000).

${ }^{42}$ M. Kato, M. Hada, R. Fukuda, and H. Nakatsuji, Chem. Phys. Lett. 408(13), 150 (2005).

${ }^{43}$ L. Visscher, T. Enevoldsen, T. Saue, H. J.A. Jensen, and J. Oddershede, J. Comput. Chem. 20(12), 1262 (1999).

${ }^{44}$ H. M. Quiney, H. Skaane, and I. P. Grant, Chem. Phys. Lett. 290, 473 (1998).

${ }^{45}$ DIRAC, a relativistic ab initio electronic structure program, Release DIRAC10 (2010), written by T. Saue, L. Visscher, and H. J.Aa. Jensen, with contributions from R. Bast, K. G. Dyall, U. Ekström, E. Eliav, T. Enevoldsen, T. Fleig, A. S. P. Gomes, J. Henriksson, M. Iliaš, Ch. R. Jacob, S. Knecht, H. S. Nataraj, P. Norman, J. Olsen, M. Pernpointner, K. Ruud, B. Schimmelpfennig, J. Sikkema, A. Thorvaldsen, J. Thyssen, S. Villaume, S. Yamamoto (see http://dirac.chem.vu.nl).

${ }^{46}$ M. Hanni, P. Lantto, M. Iliaš, H. J.A. Jensen, and J. Vaara, J. Chem. Phys. 127(16), 164313 (2007).

${ }^{47}$ M. Pecul, T. Saue, K. Ruud, and A. Rizzo, J. Chem. Phys. 121, 3051 (2004).

${ }^{48}$ A. Antušek, M. Pecul, and J. Sadlej, Chem. Phys. Lett. 427(4-6), 281 (2006).

${ }^{49}$ V. G. Malkin, O. L. Malkina, R. Reviakine, A. V. Arbuznikov, M. Kaupp, B. Schimmelpfennig, I. Malkin, M. Repiský, S. Komorovský, P. Hrobárik, E. Malkin, T. Helgaker, and K. Ruud, ReSpect program, version 3.1, 2007.

${ }^{50}$ P. Manninen, P. Lantto, J. Vaara, and K. Ruud, J. Chem. Phys. 119(5), 2623 (2003).

${ }^{51}$ C. J. Jameson, D. N. Sears, and A. C. de Dios, J. Chem. Phys. 118(6), 2575 (2003).

${ }^{52}$ W. Kutzelnigg, J. Chem. Phys. 126(20), 201103 (2007).

${ }^{53}$ L. Visscher, P. J. C. Aerts, O. Visser, and W. C. Nieuwpoort, Int. J. Quantum Chem., Quantum Chem. Symp. 40(S25), 131 (1991).

${ }^{54}$ W. Kutzelnigg, Int. J. Quantum Chem. 25(1), 107 (1984).

${ }^{55}$ K. G. Dyall, J. Chem. Phys. 100(3), 2118 (1994). 
${ }^{56}$ W. Kutzelnigg and W. Liu, J. Chem. Phys. 123(24), 241102 (2005).

${ }^{57}$ L. Visscher and T. Saue, J. Chem. Phys. 113(10), 3996 (2000).

${ }^{58}$ K. Schwarzschild, Gött. Nach. Math.-Phys. Kl. 128, 126 (1903).

${ }^{59}$ M. Gell-Mann, Nuovo Cimento, Suppl. 4, 848 (1956).

${ }^{60}$ T. Saue and T. Helgaker, J. Comput. Chem. 23(8), 814 (2002).

${ }^{61}$ T. Saue and H. J. A. Jensen, J. Chem. Phys. 118(2), 522 (2003).

${ }^{62}$ P. Sałek, T. Helgaker, and T. Saue, Chem. Phys. 311(1-2), 187 (2005).

${ }^{63}$ R. Bast, H. J.A. Jensen, and T. Saue, Int. J. Quantum Chem. 109(10), 2091 (2009).

${ }^{64}$ P. Salek, O. Vahtras, T. Helgaker, and H. Ågren, J. Chem. Phys. 117(21), 9630 (2002).

${ }^{65}$ J. Olsen, K. L. Bak, K. Ruud, T. Helgaker, and P. Jørgensen, Theor. Chem. Acc. 90(5-6), 421 (1995), see www.springerlink.com/content/ rx2frduqqx $57 \mathrm{c} 9 \mathrm{un} /$.

${ }^{66}$ L. Visscher, Theor. Chem. Acc. 98, 68 (1997).

${ }^{67}$ DALTON, a molecular electronic structure program, Release Dalton2011 (2011), see http://daltonprogram.org/.

${ }^{68} \mathrm{~K}$. Huber and G. Herzberg, Molecular Spectra and Molecular Structure. IV. Constants of Diatomic Molecules (Van Nostrand Reinhold, New York, 1979). See also the NIST web site: http://webbook.nist.gov/.

${ }^{69}$ J. Styszynski, Chem. Phys. Lett. 317(3-5), 351 (2000).

${ }^{70}$ L. Visscher and K. G. Dyall, At. Data Nucl. Data Tables 67(2), 207 (1997). See also http://www.few.vu.nl/visscher/FiniteNuclei/FiniteNuclei.htm.

${ }^{71}$ J. C. Slater, Phys. Rev. 81(3), 385 (1951).

${ }^{72}$ S. H. Vosko, L. Wilk, and M. Nusair, Can. J. Phys. 58, 1200 (1980).

${ }^{73}$ J. P. Perdew, Phys. Rev. B. 33(12), 8822 (1986); 34, 7406 (1986).

${ }^{74}$ J. P. Perdew and W. Yue, Phys. Rev. B. 33(12), 8800 (1986); 40, 3399 (1989).

${ }^{75}$ A. D. Becke, Phys. Rev. A. 38(6), 3098 (1988).

${ }^{76}$ A. D. Becke, J. Chem. Phys. 98(7), 5648 (1993).

${ }^{77}$ T. W. Keal and D. J. Tozer, J. Chem. Phys. 119(6), 3015 (2003).

${ }^{78}$ D. E. Woon and T. H. Dunning, J. Chem. Phys. 98(2), 1358 (1993).
${ }^{79}$ T. H. Dunning, J. Chem. Phys. 90(2), 1007 (1989).

${ }^{80}$ K. G. Dyall, Theor. Chem. Acc. 109, 335 (2003). The basis sets are available from the dirac web site http://dirac.chem.sdu.dk.

${ }^{81}$ K. G. Dyall, Theor. Chem. Acc. 115, 441 (2006). The basis sets are available from the dirac web site http://dirac.chem.sdu.dk.

${ }^{82}$ J. Vaara and P. Pyykkö, J. Chem. Phys. 118, 2973 (2003).

${ }^{83}$ A. Wu, Y. Zhang, X. Xu, and Y. Yan, J. Comput. Chem. 28, 2431 (2007).

${ }^{84}$ T. Helgaker, P. J. Wilson, R. D. Amos, and N. C. Handy, J. Chem. Phys. 113(8), 2983 (2000).

${ }^{85}$ G. Magyarfalvi and P. Pulay, J. Chem. Phys. 119, 1350 (2003).

${ }^{86}$ T. W. Keal and D. J. Tozer, J. Chem. Phys. 121(12), 5654 (2004).

${ }^{87}$ T. W. Keal, D. J. Tozer, and T. Helgaker, Chem. Phys. Lett. 391, 374 (2004).

${ }^{88}$ L. Armangue, M. Sola, and M. Swart, J. Phys. Chem. A. 115(7), 1250 (2011).

${ }^{89}$ W. G. Schneider, H. J. Bernstein, and J. A. Pople, J. Chem. Phys. 28(4), 601 (1958).

${ }^{90}$ W. T. Raynes, Nuclear Magnetic Resonance, Specialist Periodical Report Vol. 7 (The Royal Society of Chemistry, Cambridge, 1978).

${ }^{91}$ D. B. Chesnut, Ab Initio Calculations of NMR Chemical Shielding, Annual Reports on NMR Spectroscopy Vol. 29 (Academic Press, New York, 1994), pp. 71-122.

${ }^{92}$ A. Antušek, M. Jaszuński, and M. Olejniczak, J. Comput. Theor. Chem. 970(1-3), 54 (2011).

${ }^{93}$ O. L. Malkina, S. Komorovský, L. Visscher, and V. G. Malkin, J. Chem. Phys. 134(8), 086101 (2011).

${ }^{94}$ P. Lantto, K. Jackowski, W. Makulski, M. Olejniczak, and M. Jaszunski, J. Phys. Chem. A. 115(38), 10617 (2011).

${ }^{95}$ P.-O. Åstrand and K. V. Mikkelsen, J. Chem. Phys. 104(2), 648 (1996).

${ }^{96}$ D. K. Hindermann and C. D. Cornwell, J. Chem. Phys. 48(9), 4148 (1968).

${ }^{97}$ D. Sundholm, J. Gauss, and A. Schäfer, J. Chem. Phys. 105(24), 11051 (1996).

${ }^{98}$ T. D. Gierke and W. H. Flygare, J. Am. Chem. Soc. 94(21), 7277 (1972). 